\title{
SATISFACCIÓN FAMILIAR, \\ DEPRESIÓN Y RENDIMIENTO ACADÉMICO EN ADOLESCENTES DE UN COLEGIO ESTATAL DE HUÁNUCO, PERÚ
}

\section{FAMILY SATISFACTION, DEPRESSION AND ACADEMIC PERFORMANCE IN TEENS OF A STATE SCHOOL OF HUÁNUCO, PERÚ}

Miguel Alfredo Carrasco Muñoz', Cecilia Martínez Morales²,

Freddy Noreña Tello ${ }^{3}$, Carlos L. Bao Condor

1Universidad Nacional Hermilio Valdizán, Huánuco, Perú

$1 \quad$ Miguel Alfredo Carrasco Muñoz miguelcm.29@gmail.com https://orcid.org/0000-0002-4919-901X

2 Cecilia Martínez-Morales s, b, ${ }^{b}$ cemartinez399@gmail.com, https://orcid.org/0000-0001-6575-0556

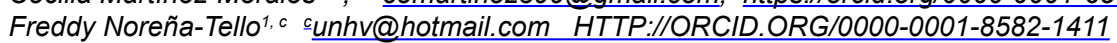

Carlos L. Bao-Condor 1, d dleodan456@hotmail.com https://orcid.org/0000-0003-1705-2221 


\section{RESUMEN}

La presente investigación tuvo como objetivo determinar la relación que existe entre Satisfacción Familiar, Depresión y Rendimiento académico en adolescentes de una Institución Educativa Secundaria de Huánuco. El tipo de investigación es no experimental y responde al Diseño Transeccional de tipo Correlacional. La población total fue de 593, para lo que se realizó el muestreo probabilístico de tipo estratificado en la que se obtuvo una muestra de 234 adolescentes del 4 to y 5 to de secundaria a los que se les administró la "Escala de Satisfacción Familiar", Inventario de Depresión de BeckIl adaptación huanuqueña (BDI-AH) y para el Rendimiento académico se consideró el registro de matrícula y el reporte de notas. Así mismo para el análisis de datos se utilizó el paquete estadístico SPSS, a través del cual para contrastar las Hipótesis específicas, se utilizó el estadístico de prueba de Chi cuadrado, por lo que con una probabilidad de error de $0,0 \%$ existe relación significativa, en consecuencia se rechaza la hipótesis nula y se acepta la hipótesis de investigación. Llegando a la conclusión que existe relación significativa entre la satisfacción familiar, depresión y rendimiento académico en adolescentes de una Institución Educativa Secundaria - Huánuco.

PALABRAS CLAVE: satisfacción familiar, depresión, rendimiento académico

\section{ABSTRACT}

The purpose of this research was to determine the relationship between Family Satisfaction, Depression and Academic Performance in adolescents of a Secondary Educational Institution of Huánuco. The type of research is non-experimental and responds to the Transectional Design of the Correlational type. The total population was 593, for which the probabilistic sampling of the stratified type was carried out in which a sample of 234 adolescents of the 4th and 5th year of secondary school was obtained to whom the "Family Satisfaction Scale", Inventory of Depression of Beck-II adaptation Huanuqueña (BDI-AH) and for the Academic Performance the registration of registration and the report of grades were considered. Likewise, for the data analysis the statistical package SPSS was used, through which to test the specific hypotheses, the Chi-square test statistic was used, so with a probability of error of $0.0 \%$ there is a significant relationship , so the null hypothesis is rejected and the research hypothesis is accepted. Coming to the conclusion that there is a significant relationship between family satisfaction, depression and academic performance in adolescents of a Secondary Educational Institution - Huánuco.

KEYWORDS: Family Satisfaction, Depression, Academic Performance

\section{INTRODUCCIÓN}

La problemática suscitados en el claustro familiar de una u otra manera tiene mucho que ver con el desenvolvimiento y desarrollo de los hijos, por lo general los acontecimientos negativos que se producen en la familia se relacionan de diversas formas con los diferentes niveles de vida de los adolescentes. En este sentido cabe señalar que el estado psicológico como la depresión no es ajeno a los acontecimientos familiares, así como tampoco su desempeño académico.

Por otra parte, hay que tener en consideración que la familia es la base para el desarrollo integral del ser humano, donde los padres cumplen un rol importante y quizás determinante en la salud psicológica y la formación educacional de los futuros ciudadanos.

El presente estudio nos permitió obtener resultados sobre qué tan satisfechos están los adolescentes en su entorno familiar, si existen indicadores de depresión en ellos a consecuencia de su relación familiar y como estas influyen en 
su rendimiento académico, teniendo en cuenta también consideraciones como son: lugar de procedencia es decir la zona en donde viven ya sea rural, urbano o urbano marginal, estructura familiar como son nuclear, monoparental o extensa. También, de los resultados de esta investigación se obtuvo información que es de utilidad para la Institución Educativa en la que se realizó el estudio, es decir en función a la muestra, en quienes se podrá intervenir de acuerdo a los resultados, de forma individual y grupal tanto con los padres así como también con los propios estudiantes, mejorando así su relación familiar ya que esta es importante para el buen desarrollo de sus integrantes, también los estudiantes que se detecten con depresión puedan tener un tratamiento adecuado para superar este terrible trastorno ya que se ha hecho cada vez prevalente en nuestra sociedad, requiriendo estas medidas de intervención, promoción y prevención urgente, ya que es este un problema de salud pública que afectan no solo a adolescente sino también en otras etapas de la vida, por ultimo intervenir de manera familiar para que los adolescentes puedan mejorar su rendimiento académico si estas fuesen afectadas por la relación familiar o por la depresión. Por todo ello es que consideramos importante este estudio, y especialmente porque se orienta a abordar dicha problemática en un grupo vulnerable como son los adolescentes.

La Organización Mundial de la Salud (2018). Define la adolescencia como el periodo de crecimiento y desarrollo humano que se produce después de la niñez y antes de la edad adulta, entre los 10 y los 19 años. Tratándose esta de una de las etapas del desarrollo del ser humano, que se caracteriza por una serie de cambios determinantes biológicos siendo esta de manera universal, pero estas pueden variar el periodo de duración y las características ligadas a este periodo pueden variar entre unas y otras culturas y según el contexto socioeconómico. Se entiende también a la adolescencia como un periodo de transición, una etapa del ciclo de crecimiento que marca el final de la niñez y prenuncia la adultez, donde se inician muchos cambios como: puberales que se caracteriza por transformaciones biológicas, psicológicas y sociales, que pueden generar crisis y conflictos que para muchos jóvenes la adolescencia es un periodo de incertidumbre e inclusive de desesperación; para otros, es una etapa de amistades internas, de aflojamiento de ligaduras con los padres, y de sueños acerca del futuro. (Urquizo \& Vela, 2017).

El significado etimológico de la palabra "familia" es bastante disperso; así encontramos que, proviene del latín "fammes" que significa hambre, y alude al hecho de que es en el grupo del seno doméstico donde el hombre satisface sus necesidades primarias. Otra postura es que proviene de la voz "fammulus" que significa siervo, aludiendo al hecho de que las familias de Roma, incluía a gente de condición servil, como esclavos, clientes, o donde los miembros del sistema familiar estaban servilmente sometidos a la autoridad del "pater" (Sobrino, 2008).

Según Quiroz (2018) la familia es un sistema abierto en constante interacción con los entornos histórico, social, económico y cultural; no es una unidad homogénea en su conformación, lo cual indica que no todas están integradas de igual manera. Por tanto, hay una gran variedad de ellas; la prevalencia de los distintos tipos de familias, sus características sociodemográficas y las formas de organización hogareña y familiar varía con el tiempo y según las transformaciones económicas, demográficas y culturales del contexto social.

La Satisfacción Familiar se entiende como un conjunto de sentimientos que se evidencian en las personas al estar con su familia, que son el resultado de una serie de interacciones positivas, nutritivas y gratificantes que se establecen y se mantienen al interior de ella. Estas relaciones son vividas de manera propia y 
única en cada miembro de la familia. (Quezada, Zavala y Lenti, 2015). Cabe destacar que el grado de satisfacción familiar percibido por el/ la adolescente se encontrará inevitablemente en función de sus necesidades personales y de la experiencia previa acumulada respecto a su realidad familiar. (Sandoval \& Sepúlveda, 2012).

Sotil y Quintana, (2003) y Condori, (2002), como se citó en Cristóbal (2017), realizó diversas investigaciones para describir y medir la dinámica familiar, es así que construye el Modelo Circunflejo donde plantea tres dimensiones centrales del comportamiento familiar:

La cohesión: Se define como el vínculo emocional que los miembros de la familia tienen entre miembros de la familia están separados o conectados a ella. Dentro del modelo circumplejo, los conceptos específicos para medir y diagnosticar la dimensión de cohesión familiar son: La vinculación emocional, los límites, las coaliciones, el tiempo, el espacio, los amigos, la toma de decisiones, el interés y las recreaciones.

La adaptabilidad familiar: Tiene que ver con la medida en que el sistema familiar es flexible y capaz de cambiar. Se define como la habilidad de un sistema marital o familiar para cambiar su estructura de poder, las relaciones de roles y las reglas de las relaciones, en respuesta al estrés situacional y propio del desarrollo. Los conceptos específicos para diagnosticar y medir la dimensión de adaptabilidad son el poder en la familia (asertividad, control, disciplina), el estilo de negociación, las relaciones de roles y las reglas de las relaciones.

Según Lafosse. como se citó en Meza (2010), Al estudiar a la familia peruana es difícil encontrar algunas características únicas ya que la realidad familiar en muy diversa. Dentro de esta diversidad están las familias que tienen una base matrimonial; las de tipo convivencial, también las familias incompletas debido a rupturas conyugales viudez, etc. Esto varía de acuerdo a la región geográfica, el nivel cultural y económico, factores que se relacionan generando problemáticas específicas y concretas. Para Quiroz (2018) entendemos como Ciclo Vital de la Familia (CVF) o Ciclo Evolutivo Familiar, a la secuencia de estadios por los que atraviesa la familia desde su establecimiento hasta su disolución. Este ciclo está constituido por etapas de complejidad creciente a las que siguen otras de simplificación familiar; además, las características sociales y económicas varían desde su formación hasta su disolución. También hay que tener en cuenta la dinámica familiar ya que es un proceso en el que intervienen interacciones, transacciones, sentimientos, pautas de conducta, expectativas, motivaciones y necesidades entre los integrantes de una familia; este proceso se efectúa en un contexto cambiante, en cada etapa evolutiva familiar y permite o no la adaptación, el crecimiento, desarrollo, madurez y funcionamiento del grupo familiar (Gómez, Irigores y Ponce, 2005).

Encontramos que, para la Organización Mundial de la Salud, la Depresión es definida como un trastorno mental frecuente, que se caracteriza por la presencia de tristeza, pérdida de interés o placer, sentimientos de culpa o falta de autoestima, trastornos del sueño o del apetito, sensación de cansancio y falta de concentración. (Cisneros \& Mantilla, 2016).

Según García-Allen (2018) para Beck, los trastornos psicológicos derivan de distorsiones cognitivas (errores en los procesos cognitivos), que son maneras equivocadas de pensar que aparecen en forma de pensamientos automáticos (productos cognitivos) ante determinadas situaciones, y que provocan estados emocionales negativos y conductas inadecuadas. Por tanto, estas distorsiones cognitivas son provocadas por creencias irracionales 0 supuestos personales aprendidos en el pasado, que 
condicionan inconscientemente la percepción e interpretación del pasado, presente y futuro.

También los niños y adolescentes pueden tener dificultad en describir e identificar sus emociones y sentimientos. Muchas veces no saben cómo comunicarse con palabras y muestran sus emociones en su comportamiento. (MINSA, 2007).

Al respecto Lazo (2015) nos dice que, durante la adolescencia se experimenta un estado depresión es común, debido a los cambios hormonales que se producen en el interior del organismo o por procesos naturales de maduración, sumado a esto el estrés, conflictos con los padres o amigos la depresión puede ser una respuesta temporal a estas situaciones. Pero también la etnopsiquiatría y la etnopsicología afirman la universalidad de la depresión, pero en cada cultura se articula un lenguaje diferencial de síntomas, a través de los cuales se expresa la enfermedad. (Aguirre, 2008). Por lo tanto, es necesario tener en cuenta las circunstancias culturales en la que se desarrolla la persona para un buen diagnóstico.

La depresión es el trastorno mental más frecuente en el país. Se estima que 1'700,000 personas la padecen en el Perú, y aunque el número de casos no deja de crecer en todas las edades, la mayor parte de la población no lo ubica como un problema grave, pese a que sus efectos pueden ir desde un daño neuronal hasta la muerte a causa del suicidio. (Garay, 2016).

En el Perú, la cifra de ideación suicida entre adolescentes es más elevada que la tasa mundial $y$, según Pomalima como se citó en Sausa (2018), se explicaría por la creciente violencia familiar, pues en la mayoría de casos (entre $60 \%$ y $70 \%$ ) se ha detectado que la causa de dichas ideas tiene como base los problemas que se presentan con los padres.
Según, Figueroa, como se citó en Huamaní \& Limaco (2017), manifiesta que el Rendimiento académicocomo"elconjuntodetransformaciones operadas en el educando, a través del proceso enseñanza-aprendizaje, que se manifiesta mediante el crecimiento y enriquecimiento de la personalidad en formación". De esta afirmación se puede sustentar, que el Rendimiento académico, no sólo son las calificaciones que el estudiante obtiene mediante pruebas $u$ otras actividades, sino que también influye su desarrollo y madurez biológica y psicológica. Podemos decir que de la misma manera que cada trabajo tiene su remuneración, así la del estudiante son las calificaciones.

El reporte oficial. MINEDU 2013, como se citó en Muelle (2016), observa que los resultados de PISA 2012 reflejan un sistema educativo que no asegura a la gran mayoría de estudiantes el logro académico suficiente en las tres competencias evaluadas, que la excelencia académica es prácticamente inexistente $\mathrm{y}$, tal como también lo revelan las evaluaciones nacionales realizadas desde 1996 y otros estudios, los autores solicitan mayor acción y compromiso de padres, docentes y directores y también de las instancias de gobierno para implementar políticas realmente efectivas. La atención de los estudiantes considera los ritmos, estilos y niveles de aprendizaje, así como su pluralidad lingüística y cultural. En este nivel, se tienen en cuenta los riesgos a los que los púberes y adolescentes están expuestos y que pueden interrumpir su escolaridad, con la finalidad de tomar medidas preventivas y pertinentes según sus características y necesidades. En ese sentido, es vital el trabajo permanente y coordinado entre los estudiantes, las familias, los directivos y los docentes (MINEDU, 2017).

\section{MATERIAL Y MÉTODOS}

El tipo de estudio fue Descriptivo-Correlacional; de diseño Transeccional-correlacional (Hernández, Fernández y Baptista, 2006). 
La población de estudio está conformada por todos los participantes del 4to y 5to grado académico, que constituyen un total de 593 alumnos varones y mujeres de nivel de Educación Secundaria de una Institución Educativa de secundaria - Huánuco 2018.

\section{MUESTRA (PARTICIPANTES)}

El muestreo que se consideró para el presente estudio es probabilístico, de tipo estratificado. La muestra está conformada por un total de 234 estudiantes de sexo masculino (89) y femenino (145) cuyas edades fluctúan entre los 14 a 19 años, matriculados en el año lectivo 2018, pertenecientes a los grados 4to y 5 to de secundaria que se encuentran distribuidos entre las secciones "A" a la "J".

\section{INSTRUMENTOS}

Para lograr los objetivos de investigación se aplicaron de manera simultánea los siguientes instrumentos de medición:

\section{Cuestionario de Satisfacción Familiar} - CSF de Olson y Wilson (1982): es un cuestionario que mide en primer lugar, medir la satisfacción familiar en las dimensiones de cohesión y adaptabilidad familiar. Los ítems correspondientes a la sub escala de cohesión familiar son los siguientes: 1, 3, 5, 7, 9, 11, 13 y 14. Los ítems correspondientes a la sub escala de adaptabilidad familiar son los siguientes: 2, 4, 6, 8, 10 y 12 .

Administración: es individual y colectiva y su aplicación dura aproximadamente entre 8 a 10 minutos.

Calificación: se da a través de la puntuación valorada para cada ítem de 1 a 5 puntos considerando 1Insatisfecho, 2 Algo Insatisfecho, 3 En General Satisfecho, 4 Muy Satisfecho y 5 Extremadamente Satisfecho. La suma de los puntajes en la Escala General es: puntaje entre $0-13$ corresponde a uno nivel de Satisfacción Insatisfecho; puntaje entre 14 - 28 corresponde a un nivel de Satisfacción Algo Insatisfecho; puntaje entre 29 - 42 corresponde a un nivel de Satisfacción En General Satisfecho; puntaje entre $43-56$ corresponde a un nivel de Satisfacción Muy Satisfecho y puntaje entre 57 - 70 corresponde a un nivel de Satisfacción Extremadamente Satisfecho. En cuanto a la Dimensión Cohesión Familiar: puntaje entre $0-8$ corresponde a uno nivel de Satisfacción Insatisfecho; puntaje entre 9 - 16 corresponde a un nivel de Satisfacción Algo Insatisfecho; puntaje entre $17-24$ corresponde a un nivel de Satisfacción En General Satisfecho; puntaje entre $25-32$ corresponde a un nivel de Satisfacción Muy Satisfecho y puntaje entre $33-40$ corresponde a un nivel de Satisfacción Extremadamente Satisfecho. Por último, la Dimensión Adaptabilidad Familiar puntaje entre $0-6$ corresponde a uno nivel de Satisfacción Insatisfecho; puntaje entre 7 12 corresponde a un nivel de Satisfacción Algo Insatisfecho; puntaje entre $13-18$ corresponde a un nivel de Satisfacción En General Satisfecho; puntaje entre $19-24$ corresponde a un nivel de Satisfacción Muy Satisfecho y puntaje entre $25-30$ corresponde a un nivel de Satisfacción Extremadamente Satisfecho.

$\checkmark$ Inventario de depresión de Beck-II adaptación huanuqueña (BDI - AH): de Aarón T. Beck, Robert A. Steer y Gregory K. Brown. El inventario posee un total de 21 ítems.

Administración: es individual y colectiva y su aplicación dura aproximadamente entre 5 a 10 minutos. La calificación se da a través de la puntuación valorada para cada ítem de 0 a 3 puntos considerando las elecciones múltiples para un ítem, se utiliza la alternativa con el valor más alto. 
Calificación: la puntuación total máxima es 63. Se debe prestar especial atención a la puntuación correcta de Cambios de Hábitos (ítem 16) y Cambios de Apetito (ítem 18). Cada uno de estos ítems contiene siete opciones con valores ordenados $(0,1 \mathrm{a} .1 \mathrm{~b}$, $\left.2 a, 2 b, 3^{a}, 3 b\right)$ que sirven para diferenciar entre incrementos y disminuciones del comportamiento y/o de la motivación. La puntuación total obtenida debe ser traducida a categorías de depresión, correspondiente a la categoría Mínimo: puntaje entre de 0 al 17; correspondiente a la categoría Leve: puntaje entre 08 al 11; correspondiente a la categoría Moderada: puntaje entre 12 al 17; correspondiente a la categoría Severo: puntaje entre 18 al 63.

$\checkmark$ Rendimiento académico: La fuente que se utilizó con respecto al rendimiento académico fue a través del Registro de matrícula y el
Reporte de notas de fin de año (Promedio de notas) de los alumnos del 4to y 5 to grado de secundaria del año académico 2018, proporcionado por el centro de estudios. Así mismo, con respecto al rendimiento académico se tomó en cuenta el promedio de notas en cuya codificación se consideró los siguientes niveles: Alto: 18 - 20, Medio: 14 - 17, Bajo: 11 - 13 y Deficiente: 00 - 10

\section{RESULTADOS}

En relación al Género y satisfacción familiar en adolescentes de una Institución Educativa Secundaria - Huánuco, se muestra en la Tabla $\mathrm{N}^{\circ} 01$ donde se puede apreciar la satisfacción familiar y género; en la cual el género femenino presenta mayor cantidad de adolescentes muy satisfechos siendo estas el 24,4\%(57) de la muestra total; en tanto el género masculino presenta menor cantidad adolecentes insatisfechos con un 0,4\%(1) de la muestra total.

Tabla $\mathrm{N}^{\circ} 01$

Género y satisfacción familiar en adolescentes de una Institución Educativa Secundaria - Huánuco

\begin{tabular}{|c|c|c|c|c|c|c|c|c|c|c|c|c|}
\hline \multirow{3}{*}{ GÉNERO } & \multicolumn{10}{|c|}{ SATISFACIÓN FAMILIAR } & \multirow{2}{*}{\multicolumn{2}{|c|}{ Total }} \\
\hline & \multicolumn{2}{|c|}{ INSATISFECHO } & \multicolumn{2}{|c|}{$\begin{array}{c}\text { ALGO } \\
\text { INSATISFECHO }\end{array}$} & \multicolumn{2}{|c|}{$\begin{array}{c}\text { EN } \\
\text { GENERAL } \\
\text { SATISFECHO }\end{array}$} & \multicolumn{2}{|c|}{$\begin{array}{c}\text { MUY } \\
\text { SATISFECHO }\end{array}$} & \multicolumn{2}{|c|}{$\begin{array}{l}\text { EXTREMADAMENTE } \\
\text { SATISFECHO }\end{array}$} & & \\
\hline & $\mathrm{fi}$ & $\%$ & $\mathrm{fi}$ & $\%$ & fi & $\%$ & $\mathrm{fi}$ & $\%$ & $\mathrm{fi}$ & $\%$ & fi & $\%$ \\
\hline MASCULINO & 1 & 0,4 & 7 & 3,0 & 20 & 8,5 & 37 & 15,8 & 24 & 10,3 & 89 & 38,0 \\
\hline FEMENINO & 2 & 0,9 & 11 & 4,7 & 51 & 21,8 & 57 & 24,4 & 24 & 10,3 & 145 & 62,0 \\
\hline Total & 3 & 1,3 & 18 & 7,7 & 71 & 30,3 & 94 & 40,2 & 48 & 20,5 & 234 & 100,0 \\
\hline
\end{tabular}

Fuente: Administración de la "Escala de Satisfacción familiar"

En la Tabla $\mathrm{N}^{\circ} 02$ se puede apreciar la depresión y género; en la cual el género femenino presenta mayor cantidad de adolescentes con depresión mínimo siendo estas el 20,9\%(49) de la muestra total; en tanto el género masculino presenta menor cantidad de adolescentes con depresión severo con un $6,4 \%(15)$ de la muestra total. 
Tabla $\mathrm{N}^{\circ} 02$

Género y depresión en adolescentes de una Institución Educativa Secundaria - Huánuco

\begin{tabular}{|c|c|c|c|c|c|c|c|c|c|c|}
\hline & \multicolumn{9}{|c|}{ DEPRESIÓN } & \multicolumn{2}{c|}{ Total } \\
\cline { 2 - 11 } & \multicolumn{2}{|c|}{ MINIMO } & \multicolumn{2}{|c|}{ LEVE } & \multicolumn{2}{|c|}{ MODERADO } & \multicolumn{2}{c|}{ SEVERO } & \multicolumn{2}{c|}{} \\
\cline { 2 - 11 } & & & & & & & & & & \\
GÉNERO & & & $\mathrm{fi}$ & $\%$ & $\mathrm{fi}$ & $\%$ & $\mathrm{fi}$ & $\%$ & $\mathbf{f i}$ & $\%$ \\
\hline MASCULINO & 36 & 15,4 & 20 & 8,5 & 18 & 7,7 & 15 & 6,4 & $\mathbf{8 9}$ & $\mathbf{3 8 , 0}$ \\
\hline FEMENINO & 49 & 20,9 & 34 & 14,5 & 23 & 9,8 & 39 & 16,7 & $\mathbf{1 4 5}$ & $\mathbf{6 2 , 0}$ \\
\hline Total & $\mathbf{8 5}$ & $\mathbf{3 6 , 3}$ & $\mathbf{5 4}$ & $\mathbf{2 3 , 1}$ & $\mathbf{4 1}$ & $\mathbf{1 7 , 5}$ & $\mathbf{5 4}$ & $\mathbf{2 3 , 1}$ & $\mathbf{2 3 4}$ & $\mathbf{1 0 0 , 0}$ \\
\hline
\end{tabular}

Fuente: Administración del Inventario de depresión de Beck II

En la tabla $\mathrm{N}^{\circ} 03$ se puede apreciar el rendimiento académico y género; en la cual el género femenino presenta mayor cantidad de adolescentes con rendimiento académico alto siendo estas el $11,1 \%(26)$ de la muestra total; en tanto el género masculino presenta menor cantidad de adolescentes con rendimiento académico deficiente con un $1,3 \%(3)$ de la muestra total.

Tabla $\mathrm{N}^{\circ} 03$

Género y rendimiento académico en adolescentes de una Institución Educativa Secundaria - Huánuco

\begin{tabular}{|c|c|c|c|c|c|c|c|c|c|c|}
\hline & \multicolumn{9}{|c|}{ RENDIMIENTO ACADÉMICO } & \multicolumn{3}{|c|}{ Total } \\
\cline { 2 - 12 } & \multicolumn{2}{|c|}{ DEFICIENTE } & \multicolumn{2}{|c|}{ BAJO } & \multicolumn{2}{|c|}{ MEDIO } & \multicolumn{2}{|c|}{ ALTO } & \multicolumn{2}{|c|}{} \\
\cline { 2 - 11 } GÉNERO & $\mathrm{fi}$ & $\%$ & $\mathrm{fi}$ & $\%$ & $\mathrm{fi}$ & $\%$ & $\mathrm{fi}$ & $\%$ & $\mathbf{f i}$ & $\%$ \\
\hline MASCULINO & 3 & 1,3 & 20 & 8,5 & 52 & 22,2 & 14 & 6,0 & $\mathbf{8 9}$ & $\mathbf{3 8 , 0}$ \\
\hline FEMENINO & 9 & 3,8 & 33 & 14,1 & 77 & 32,9 & 26 & 11,1 & $\mathbf{1 4 5}$ & $\mathbf{6 2 , 0}$ \\
\hline Total & $\mathbf{1 2}$ & $\mathbf{5 , 1}$ & $\mathbf{5 3}$ & $\mathbf{2 2 , 6}$ & $\mathbf{1 2 9}$ & $\mathbf{5 5 , 1}$ & $\mathbf{4 0}$ & $\mathbf{1 7 , 1}$ & $\mathbf{2 3 4}$ & $\mathbf{1 0 0 , 0}$ \\
\hline
\end{tabular}

Fuente: Registro de matrícula y Reporte de notas

Al realizar el análisis inferencial de la correlación de la Hipótesis Especifica 1 que corresponde a la relación entre satisfacción familiar y rendimiento académico en adolescentes de una Institución Educativa Secundaria - Huánuco, contrastar las hipótesis se utiliza el estadístico de prueba de Chi cuadrado; el nivel de confianza es del
$95 \%$ y el error alfa es de $5 \%$. El chi ${ }^{2}$ calculado es de 96,2 para 12 grados de libertad $(21,03)$, el $p$ valor es de $0.000(p<0,05)$ el que está por debajo del error alfa establecido; por lo que con una probabilidad de error de $0,0 \%$ existe relación significativa entre la satisfacción familiar y rendimiento académico en adolescentes de una 
Institución Educativa Secundaria - Huánuco,

como se puede apreciar en la tabla $\mathrm{N}^{\circ} 04$.

Tabla $\mathrm{N}^{\circ} 04$

Relación entre satisfacción familiar y rendimiento académico en adolescentes de una Institución Educativa Secundaria - Huánuco

\begin{tabular}{|c|c|c|c|c|c|c|c|c|c|c|}
\hline \multirow{3}{*}{$\begin{array}{l}\text { SATISFACIÓN } \\
\text { FAMILIAR }\end{array}$} & \multicolumn{8}{|c|}{ RENDIMIENTO ACADÉMICO } & \multirow{2}{*}{\multicolumn{2}{|c|}{ Total }} \\
\hline & \multicolumn{2}{|c|}{ DEFICIENTE } & \multicolumn{2}{|c|}{ BAJO } & \multicolumn{2}{|c|}{ MEDIO } & \multicolumn{2}{|c|}{ ALTO } & & \\
\hline & $\mathrm{fi}$ & $\%$ & $\mathrm{fi}$ & $\%$ & $\mathrm{fi}$ & $\%$ & $\mathrm{fi}$ & $\%$ & $\mathrm{fi}$ & $\%$ \\
\hline INSATISFECHO & 2 & 0,9 & 0 & 0,0 & 1 & 0,4 & 0 & 0,0 & 3 & 1,3 \\
\hline $\begin{array}{l}\text { ALGO } \\
\text { INSATISFECHO }\end{array}$ & 6 & 2,6 & 9 & 3,8 & 3 & 1,3 & 0 & 0,0 & 18 & 7,7 \\
\hline $\begin{array}{l}\text { EN GENERAL } \\
\text { SATISFECHO }\end{array}$ & 3 & 1,3 & 20 & 8,5 & 44 & 18,8 & 4 & 1,7 & 71 & 30,3 \\
\hline MUY SATISFECHO & 1 & 0,4 & 17 & 7,3 & 59 & 25,2 & 17 & 7,3 & 94 & 40,2 \\
\hline $\begin{array}{l}\text { EXTREMADAMENTE } \\
\text { SATISFECHO }\end{array}$ & 0 & 0,0 & 7 & 3,0 & 22 & 9,4 & 19 & 8,1 & 48 & 20,5 \\
\hline Total & 12 & 5,1 & 53 & 22,6 & 129 & 55,1 & 40 & 17,1 & 234 & 100,0 \\
\hline $\mathrm{Chi}^{2}$ & & & & & & & & $P$ val & & \\
\hline 96,2 & & & & & & & & 0,00 & & \\
\hline
\end{tabular}

Fuente: Base de datos de los instrumentos de medición

Para la correlación de la Hipótesis especifica 2 que corresponde a la relación se utiliza el estadístico de prueba de Chi cuadrado; el nivel de confianza es del $95 \%$ y el error alfa es de $5 \%$. El chi ${ }^{2}$ calculado es de 87,3 para 9 grados de libertad $(16,92)$, el $p$ valor es de $0.000(p<0,05)$ el que está por debajo del error alfa establecido; por lo que con una probabilidad de error de $0,0 \%$ existe relación significativa entre depresión y rendimiento académico en adolescentes de una Institución Educativa Secundaria - Huánuco, como se puede apreciar en la tabla $\mathrm{N}^{\circ} 05$. 
Tabla $\mathrm{N}^{\circ} 05$

Relación entre depresión y rendimiento académico en adolescentes de una institución educativa secundaria - Huánuco

\begin{tabular}{|c|c|c|c|c|c|c|c|c|c|c|}
\hline \multirow[b]{3}{*}{ DEPRESIÓN } & \multicolumn{8}{|c|}{ RENDIMIENTO ACADÉMICO } & \multirow{2}{*}{\multicolumn{2}{|c|}{ Total }} \\
\hline & \multicolumn{2}{|c|}{ DEFICIENTE } & \multicolumn{2}{|c|}{ BAJO } & \multicolumn{2}{|c|}{ MEDIO } & \multicolumn{2}{|c|}{ ALTO } & & \\
\hline & $\mathrm{fi}$ & $\%$ & $\mathrm{fi}$ & $\%$ & fi & $\%$ & $\mathrm{fi}$ & $\%$ & fi & $\%$ \\
\hline MINIMO & 0 & 0,0 & 12 & 5,1 & 46 & 19,7 & 27 & 11,5 & 85 & 36,3 \\
\hline LEVE & 0 & 0,0 & 12 & 5,1 & 31 & 13,2 & 11 & 4,7 & 54 & 23,1 \\
\hline MODERADO & 0 & 0,0 & 6 & 2,6 & 34 & 14,5 & 1 & 0,4 & 41 & 17,5 \\
\hline SEVERO & 12 & 5,1 & 23 & 9,8 & 18 & 7,7 & 1 & 0,4 & 54 & 23,1 \\
\hline Total & 12 & 5,1 & 53 & 22,6 & 129 & 55,1 & 40 & 17,1 & 234 & 100,0 \\
\hline \multicolumn{3}{|c|}{$\mathrm{Chi}^{2}$} & \multicolumn{4}{|c|}{ GI } & \multicolumn{4}{|c|}{$\mathrm{P}$ valor } \\
\hline \multicolumn{3}{|c|}{87,3} & \multicolumn{4}{|c|}{9} & \multicolumn{4}{|c|}{0,000} \\
\hline
\end{tabular}

Fuente: Base de datos de los instrumentos de medición

Para la correlación de la Hipótesis especifica 3 que corresponde a la relación se utiliza el estadístico de prueba de Chi cuadrado; el nivel de confianza es del $95 \%$ y el error alfa es de $5 \%$. El chi ${ }^{2}$ calculado es de 95,9 para 12 grados de libertad $(21,03)$, el $p$ valor es de $0.000(p<0,05)$

Tabla $N^{\circ} 06$

Relación entre satisfacción familiar y depresión en adolescentes de una institución educativa secundaria - Huánuco

\begin{tabular}{|c|c|c|c|c|c|c|c|c|c|c|}
\hline \multirow[b]{3}{*}{ SATISFACIÓN FAMILIAR } & \multicolumn{8}{|c|}{ DEPRESIÓN } & \multirow{2}{*}{\multicolumn{2}{|c|}{ Total }} \\
\hline & \multicolumn{2}{|c|}{ MINIMO } & \multicolumn{2}{|c|}{ LEVE } & \multicolumn{2}{|c|}{ MODERADO } & \multicolumn{2}{|c|}{ SEVERO } & & \\
\hline & $\mathrm{fi}$ & $\%$ & $\mathrm{fi}$ & $\%$ & $\mathrm{fi}$ & $\%$ & $\mathrm{fi}$ & $\%$ & fi & $\%$ \\
\hline INSATISFECHO & 0 & 0,0 & 0 & 0,0 & 0 & 0,0 & 3 & 1,3 & 3 & 1,3 \\
\hline ALGO INSATISFECHO & 1 & 0,4 & 0 & 0,0 & 2 & 0,9 & 15 & 6,4 & 18 & 7,7 \\
\hline $\begin{array}{l}\text { EN GENERAL } \\
\text { SATISFECHO }\end{array}$ & 12 & 5,1 & 16 & 6,8 & 16 & 6,8 & 27 & 11,5 & 71 & 30,3 \\
\hline MUY SATISFECHO & 42 & 17,9 & 24 & 10,3 & 19 & 8,1 & 9 & 3,8 & 94 & 40,2 \\
\hline $\begin{array}{l}\text { EXTREMADAMENTE } \\
\text { SATISFECHO }\end{array}$ & 30 & 12,8 & 14 & 6,0 & 4 & 1,7 & 0 & 0,0 & 48 & 20,5 \\
\hline Total & 85 & 36,3 & 54 & 23,1 & 41 & 17,5 & 54 & 23,1 & 234 & 100,0 \\
\hline \multicolumn{2}{|l|}{$\mathrm{Chi}^{2}$} & \multicolumn{4}{|c|}{$\mathbf{G I}$} & \multicolumn{4}{|c|}{$P$ valor } & \\
\hline \multicolumn{2}{|l|}{95,9} & \multicolumn{4}{|c|}{12} & & \multicolumn{2}{|c|}{0,000} & & \\
\hline
\end{tabular}

Fuente: Base de datos de los instrumentos de medición el que está por debajo del error alfa establecido; por lo que con una probabilidad de error de $0,0 \%$ existe relación significativa entre satisfacción familiar y depresión en adolescentes de una Institución Educativa Secundaria - Huánuco, como se puede apreciar en la tabla $\mathrm{N}^{\circ} 06$. 


\section{DISCUSIÓN}

En la investigación realizada por Salazar, Veytia, Márquez, y Huitrón (2013) sobre Relación entre satisfacción con el ambiente familiar y depresión en adolescentes, cuyos resultados indican que $12.9 \%$ de adolescentes sufría depresión y $24.8 \%$ insatisfacción familiar, hallándose además una relación significativa entre ambas. Además, los adolescentes que mostraron insatisfacción familiar tuvieron 2.38 veces más posibilidad de sufrir depresión. Por otro lado, en la presente investigación se según el análisis se encontró que el chi2 calculado es de 95,9 para 12 grados de libertad $(21,03)$, el $p$ valor es de $0.000(p<0,05)$ el que está por debajo del error alfa establecido; por lo que con una probabilidad de error de $0,0 \%$ existe relación significativa entre satisfacción familiar y depresión en adolescentes de una Institución Educativa Secundaria - Huánuco.

A su vez Villamizar, Galvis y Jiménez (2013) en su investigación, los resultados obtenidos en las pruebas mostraron satisfacción familiar y promedio académico regular. Una vez obtenido los resultados en la ESFA e identificado el promedio académico se aplicó la $r$ de Pearson para encontrar el coeficiente de correlación, para ello se utilizó el SPSS. Los resultados mostraron la inexistencia de correlación entre esas dos variables, tanto en la totalidad de los participantes como por nivel de formación. Por otro lado, en la presente investigación se según el análisis se encontró que el chi2 calculado es de 96,2 para 12 grados de libertad $(21,03)$, el $p$ valor es de $0.000(p<0,05)$ que está por debajo del error alfa establecido; por lo que con una probabilidad de error de $0,0 \%$ existe relación significativa entre la satisfacción familiar y rendimiento académico en adolescentes de una Institución Educativa Secundaria - Huánuco.

También Sánchez (2013) en la investigación obtuvo como resultado que de los 78 cuestionarios se observa que existe una relación de .839 con un nivel de significancia de 0,01 (unilateral), entre las variables: participación de los padres en la educación de sus hijos y el rendimiento académico de los mismos. En la presente investigación se según el análisis se encontró que el chi2 calculado es de 96,2 para 12 grados de libertad $(21,03)$, el $p$ valor es de $0.000(p<0,05)$ el que está por debajo del error alfa establecido; por lo que con una probabilidad de error de $0,0 \%$ existe relación significativa entre la satisfacción familiar y rendimiento académico en adolescentes de una Institución Educativa Secundaria - Huánuco.

\section{CONSIDERACIONES FINALES/ CONCLUSIONES}

- El hallazgo del análisis respecto a satisfacción familiar y rendimiento académico se observa que el $8,1 \%$ (19) de los adolescentes se encuentra extremadamente satisfechos y a la vez presentan un rendimiento académico alto; así mismo se observa una minoría que un $0.9 \%$ (2) se encuentra insatisfecho y con rendimiento académico deficiente.

- En el análisis respecto a depresión y rendimiento académico se observa que el $5,1 \%$ (12) de los adolescentes se encuentran con depresión severa y a la vez presentan un rendimiento académico deficiente; así mismo se observa que un $11,5 \%$ (27) se encuentran con depresión mínima y con rendimiento académico alto.

- En el análisis respecto a satisfacción familiar y depresión se observa que el 12,8\% (30) de los adolescentes se encuentran extremadamente satisfechos y a la vez presentan depresión mínima; así mismo se observa que un $1,3 \%$ (3) se encuentran insatisfechos y a la vez presenta depresión severa.

Llegando a la conclusión que existe relación significativa entre la satisfacción familiar, 
depresión y rendimiento académico en adolescentes de una Institución Educativa Secundaria - Huánuco.

\section{REFERENCIA BIBLIOGRÁFICA}

Aguirre, A. (2008). Antropología de la depresión. Revista Mal Estar e Subjetividade.. vol.8 $N^{\circ}$ 3. ISSN 2175-3644. pp. 566567. Recuperado de: http://pepsic. bvsalud.org/scielo.php?script $=$ sci arttext\&pid=S $1518-6148200$ 8000300002

Cisneros, S. y Mantilla, M. (2016). Depresión y ansiedad en niños de colegios estatales deldistritodechorrillos(Tesisdepregrado, Universidad Ricardo Palma, Lima, Perú) Recuperado de: http://repositorio.urp. edu.pe/bitstream/ handle/urp/858/ cisneros_dsmantilla_rm $\% 5 \mathrm{~b} 1 \% 5 \mathrm{~d}$. pdf? sequence $=1 \&$ isAllowed=y

Cristóbal, E. (2017). Funcionamiento familiar y la depresión en estudiantes de la Institución Educativa Héroes de Jactay, HUÁNUCO - 2016. (Tesis de maestría, Universidad Nacional Hermilio Valdizán, Huánuco, Perú). Recuperado de: http://repositorio. unheval.edu.pe/bitstream/handle/ UNHEVAL/1707/PPIP\%2000009\%20 C89.pdf?sequence=3\&isAllowed=y

Garay, K. (14 de junio de2016). La depresión es el trastorno mental más común en Perú. El Peruano. Recuperado de: https:// elperuano.pe/noticia-la-depresiones-trastorno-mental-mas-comunperu-41872.aspx

García-Allen, J. (2018). Psicología y Mente: La Terapia Cognitiva de Aaron Beck. Madrid, España: Copyrigth. Recuperado de: https://psicologiaymente.com/ clinica/terapia-cognitiva-aaron-beck

Gómez, F., Irigores, A. y Ponce, E. (Ed.).
(2005). Elementos esenciales de la Medicina Familiar, conceptos básicos para el estudio de las Familias. Código de Bioética en Medicina Familiar. $1^{\circ}$ Reunión de Consenso Académico en Medicina Familiar de Organismos e Instituciones Educativas y de Salud. Colonia Roma Sur, México DF: Editorial Mexicana $N^{\circ} 1812$.

Hernández. R., Fernández, C. y Baptista, P. (2006). Metodología de la Investigación. 5ta Edición. Edit. Graw Hill, México.

Huamaní, G. yLimaco, R. (2017). Relaciónentreel funcionamiento familiar y el rendimiento académico en el área de comunicación de los estudiantes de quinto Grado de Educación primaria de la I.E. 0027 San Antonio Jicamarca. (Tesis de grado) Universidad Nacional De Educación Enrique Guzmán Y Valle. Lima. Perú). Recuperado de: http://repositorio.une. edu.pe/bitstream/handle/UNE/916/ TL\%20PC-Ep\%20 H83\%202017. pdf?sequence=1\&isAllowed $=y$

Lazo, V., (2015). "Manifestaciones de la depresión y rendimiento académico de los adolescentes de 15 a 19 años". (Tesis de grado, Universidad De Cuenca, Cuenca, Ecuador). Recuperado de: http://dspace.ucuenca. edu. ec/bitstream/123456789/21220/1/ Tesis.pdf

Meza, H. (2010). Funcionamiento familiar y rendimiento académico en alumnas del tercer grado de secundaria de una institución educativa del Callao. (Tesis de maestría, Universidad San Ignacio de Loyola. Lima. Perú). Recuperado de: http://repositorio.usil.edu.pe/ bitstream/123456789/1233/1/2010_ Meza_Funcionamiento\%20familiar\%20 y $\% 20$ rendimiento $\% 20$ escolar $\% 20$ 
en $\% 20$ alumnas $\% 20$ de $\% 20$ tercer $\% 20$ grado $\% 20 \mathrm{de} \% 20$ secundaria $\% 20 \mathrm{de} \% 20$ una $\% 20$ instituci $\%$ C3\%B3n\%20educativa\%20del\%20Callao.pdf

MINEDU, (2017). Educación Básica Regular. Programa curricular de Educación Secundaria. Aprobado mediante Resolución Ministerial N. ${ }^{\circ}$ 281-2016ED Modificado mediante RM Nro. 1592017-ED Lima, Perú. Recuperado de: http://www.minedu.gob.pe/curriculo/pdf/ programa-secundaria-17-abril.pdf

MINSA, (2007). Guía técnica: guía de práctica clínica en depresión. Lima, Perú Recuperado de: http://bvs.minsa.gob. pe/local/MINSA/1084_DGSP261.pdf

Muelle, L., (2016). Factores de riesgo en el bajo desempeño académico y desigualdad social en el Perú según PISA 2012. Scielo Perú. Vol. XLIII, No 79. ISSN 0252-1865, pp. 9-45. Recuperado de: http://www.scielo.org.pe/pdf/apuntes/ v43n79/a01v43n79.pdf

Organización Mundial de la Salud (2018). Salud de la madre, el recién nacido, del niño y del adolescente. Desarrollo en la adolescencia. Disponible en: http://www. who.int/maternal_child_adolescent/ topics/adolescence/dev/es

Quezada, J, Zavala, E y Lenti, M. (2015). Satisfacción Familiar En Mujeres Jóvenes. UNIFE. Av.psicol. 23(2) 2015: pp. 223 - 229. Recuperado de: http://www.unife.edu.pe/publicaciones/ revistas/psicologia/2015_2/J.Quezada. pdf

Quiroz, C., (2018). MedicosFamiliares.com: Ciclo Vital de la Familia. Creative Commons. México. Recuperado de: http://www.medicosfamiliares.com familia /ciclo-vital-de-la-familia.html
Quiroz, C., (2018). MedicosFamiliares.com: Clasificación o Tipología de la Familia. Creative Commons. México. Recuperado de: http://www.medicosfamiliares. com/ familia/clasificacion-o-tipologia-de-lafamilia.html

Salazar, Y., Veytia, M., Márquez, O., y Huitrón, G. (2013). Relación entre satisfacción con el ambiente familiar y depresión en adolescentes. Psicología y Salud, Vol. 23, Núm. 1: p. 141-148. Recuperado de: https://www.uv.mx/psicysalud/ psicysalud-23-1/231/Yareli $\quad \% 20$ Salazar\%20 \%C3\%81lvarez.pdf

Sánchez, I., (2013). Apoyo parental y rendimiento académico. (Tesis de maestría, Universidad Autónoma de Tamaulipas, México). Recuperado de: http://bibliotecadigital.tamaulipas.gob. mx/archivos/descargas/7983545d502df a507ae1275a57a61368af287051.pdf

Sandoval, F., y Sepúlveda, C., (2012). "Autopercepción del nivel de Satisfacción Familiar y Bienestar Psicológico en Adolescentes de la Ciudad de Chillán". (Tesis de grado, Universidad del BíoBío, Chillán, Chile). Recuperado de: http://repobib.ubiobio.cl/jspui/ bitstream/123456789/1424/1/ Sandoval\%20Gutierrez\%2C\%20 Felipe\%20Alberto.pdf

Sausa, M. (03 de febrero de 2018). Menores ocupan el $70 \%$ de atenciones en salud mental. Perú 21. Recuperado de: https:// peru21.pe/peru/cifras-salud-mentalperu-menores-ocupan-70-atencionesinfografia-394376

Sobrino, L. (2008). Niveles de satisfacción familiar y de comunicación entre padres e hijos AV. PSICOL. 16(1): pp. 109-137. Recuperado de: http:// 
www.unife. edu.pe/pub/revpsicologia/ sastisfaccionfamiliar.pdf

Urquizo, D. y Vela, S. (2017). Depresión en los y las adolescentes de décimo año de educación básica en la Unidad Educativa Municipal "Quitumbe", durante el periodo octubre 2016- febrero 2017. (Tesis de pregrado, Universidad Central Del Ecuador, Quito, Ecuador) Recuperado de: http://www.dspace. uce.edu.ec/bitstream/25000/9719/1/TUCE-0006-090.pdf

Villamizar, G., Galvis, J, y Jiménez, M. (2013). "Relación entre satisfacción familiar y rendimiento académico en estudiantes de psicología de la Universidad Pontificia Bolivariana Bucaramanga". Rev Psicol Hered. 8 (1-2), 2013: pp. 1125. Recuperado de: http://www.upch. edu.pe/vrinve/dugic/revistas/index. php/ $\mathrm{RPH} /$ article/view/2945 